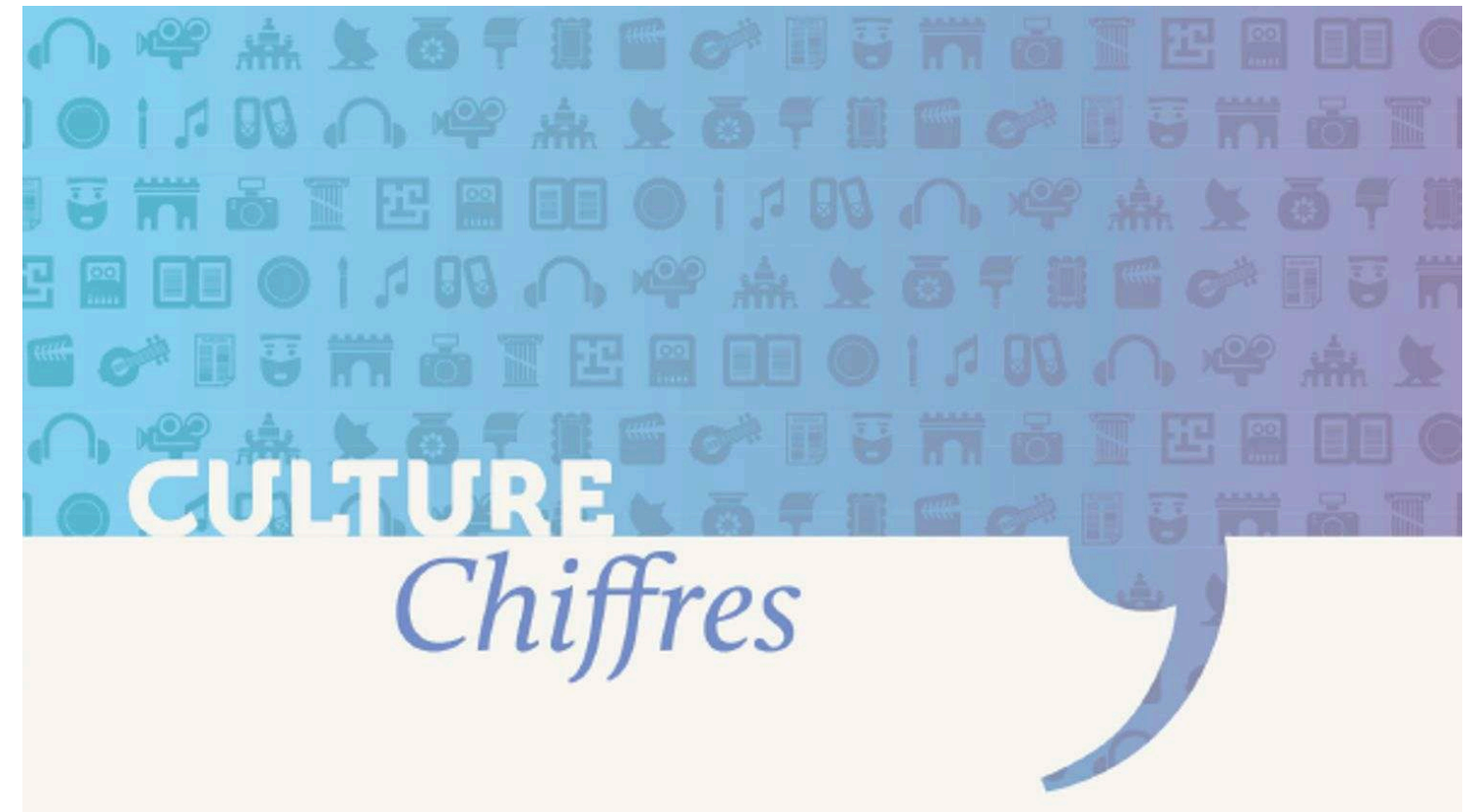

Les femmes dans

la création audiovisuelle et de spectacle vivant

Les auteurs de la SACD

percevant des droits en $201 \mathrm{II}$

Marie Gouyon

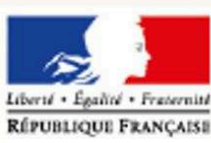

Misueve

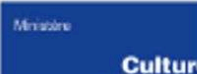

Cultur

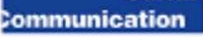

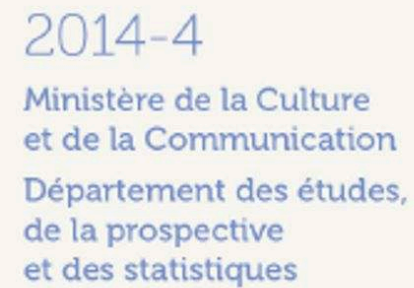

et des statistiques 


\title{
Les femmes dans la création audiovisuelle et de spectacle vivant
}

\author{
Les auteurs de la SACD percevant des droits en 2011
}

Women in audio-visual and live show creation. SACD (Society of Authors and Dramatists) authors receiving royalties in 2011

\section{Marie Gouyon}

Éditeur : Département des études, de la prospective et des statistiques

Lieu d'édition : Paris

Année d'édition : 2014

Date de mise en ligne : 21 septembre 2015

Collection : Culture chiffres

ISBN électronique : 9782111398184

\section{Sbooks}

http://books.openedition.org

\section{Édition imprimée}

Date de publication : 1 juillet 2014

Nombre de pages : 16

\section{Référence électronique}

GOUYON, Marie. Les femmes dans la création audiovisuelle et de spectacle vivant : Les auteurs de la SACD percevant des droits en 2017. Nouvelle édition [en ligne]. Paris : Département des études, de la prospective et des statistiques, 2014 (généré le 25 avril 2021). Disponible sur Internet : <http:// books.openedition.org/deps/469>. ISBN : 9782111398184. 


\section{Les femmes dans} la création audiovisuelle et de spectacle vivant

Les auteurs de la SACD

percevant des droits en $201 \mathrm{II}$

\section{Marie Gouyon}
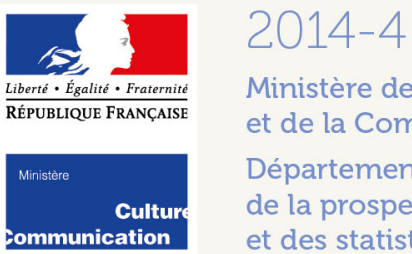

Ministère de la Culture et de la Communication

Département des études, 


\title{
Les femmes dans
}

la création audiovisuelle et de spectacle vivant

\author{
Les auteurs de la SACD \\ percevant des droits en $201 \mathrm{I}$
}

Marie Gouyon*

En 2011, les femmes représentent $29 \%$ des auteurs de la SACD et reçoivent $24 \%$ des droits versés. Une femme perçoit ainsi, en 2011, un revenu moyen inférieur de $24 \%$ à celui d'un homme.

Les écarts de droits versés entre femmes et hommes trouvent plusieurs éléments d'explication : les femmes participent à moins d'œuvres que les hommes, en particulier dans le cinéma, le théâtre et les arts de la rue. Elles n'exercent pas non plus les mêmes fonctions, et sont ainsi plus souvent auteurs de texte ou chorégraphes que réalisatrices ou compositrices.

À discipline et fonction égales, les écarts de rémunération sont pratiquement inexistants dans la radio et la télévision, mais subsistent dans le cinéma et le spectacle vivant : ces écarts persistants peuvent être liés à d'autres facteurs comme, par exemple, une fréquentation des spectacles ou une programmation des œuvres moins importante, dans des lieux de représentation ou sur des canaux de diffusion différents et à des horaires moins favorables.

\section{0 œuvres, 135 millions d'euros de droits nets répartis entre 18000 auteurs}

En 2011, 91400 œuvres audiovisuelles et de spectacle vivant, dont les droits sont gérés collectivement par la Société des auteurs compositeurs dramatiques (SACD), donnent lieu à la répartition de 135 millions d'euros de droits nets, pour leur diffusion ou leur représentation : diffusions télévisuelles des œuvres de cinéma et de télévision, diffusions d'œuvres radiophoniques, et représentations d'œuvres théâtrales, chorégraphiques, musicales, circassiennes ou d'arts de la rue (encadrés 1, 2 et 3, p. 10-13).

\footnotetext{
* DEPS.

L'analyse statistique des données relatives aux droits gérés collectivement par la Société des auteurs compositeurs dramatiques (SACD) a été produite par le DEPS. Pour leur précieuse collaboration au cours de cette étude, que soient vivement remerciés ici Pascal ROGARD, directeur général de la SACD et, au sein de la direction du développement et de la coordination de la SACD, Muriel COUTON, directrice, Violeta MATEO, directrice adjointe et, tout particulièrement, Christelle FINET, chargée d'études.
} 
Le montant des droits versés pour la diffusion d'une œuvre audiovisuelle ou la représentation d'une œuvre de spectacle vivant dépend de différents paramètres :

- pour le spectacle vivant, cela dépend du type d'œuvre (pièce de théâtre, one-manshow ou sketch par exemple pour une œuvre théâtrale), du succès du spectacle et de la capacité (en nombre de places) des lieux de représentation ;

- pour l'audiovisuel, de la durée de l'œuvre (court ou long métrage par exemple), du nombre de retransmissions, des horaires de diffusion (prime time, nuit) et des canaux de diffusion (chaînes de télévision nationales gratuites ou numériques terrestres par exemple, stations de Radio France ou autres stations de radio).

Ces droits sont versés à 18000 auteurs - qualifiés d'auteurs « actifs » (voir glossaire p. 13) - dont $29 \%$ sont des femmes (encadré 2 , p. 11). Certaines disciplines sont plus féminisées que d'autres : la danse et la radio (34\% de femmes), le théâtre (31\%), la télévision ( $29 \%$ ) et le cirque ( $28 \%$ ) le sont plus que le cinéma ( $25 \%)$, les arts de la rue (25\%) et la musique (23\%) (graphique 1$)$.

\section{Graphique 1 - Place des femmes parmi les auteurs de la SACD actifs, 2011}

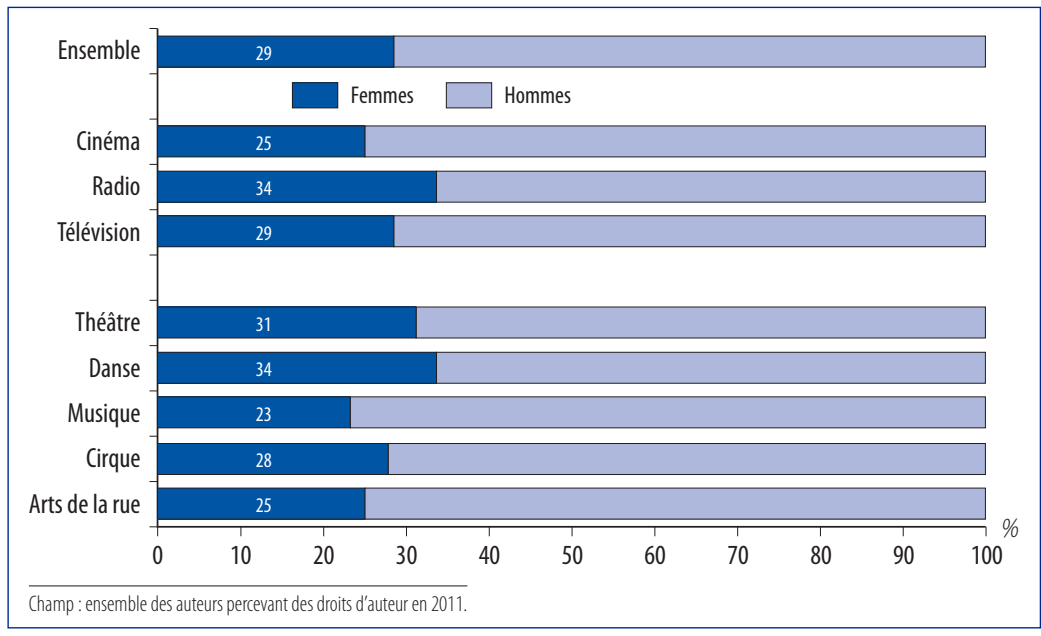

Source: SACD/DEPS, Ministère de la Culture et de Communication, 2014

En moyenne, en 2011, un auteur perçoit 7520 euros nets. L'audiovisuel est nettement plus rémunérateur (11 210 euros en moyenne) que le spectacle vivant (3 750 euros).

Les femmes représentent $29 \%$ des auteurs mais ne perçoivent que $24 \%$ des droits versés en 2011. Ainsi, en 2011, une femme auteur de l'audiovisuel ou du spectacle vivant reçoit en moyenne 6130 euros contre 8100 euros pour un homme.

L'écart de revenus entre hommes et femmes est moins accentué dans l'audiovisuel que dans le spectacle vivant. Une femme auteur de l'audiovisuel perçoit en effet, en moyenne, 10370 euros pour sa participation à la création audiovisuelle, et un homme 11520 euros. Inexistant dans la radio et la télévision, l'écart est plus marqué dans le cinéma, où le revenu annuel moyen des femmes est de $27 \%$ inférieur à celui des hommes.

Dans le spectacle vivant, ces niveaux moyens s'établissent à 2520 euros pour une femme et 4290 euros pour un homme. Par rapport au revenu des hommes, le revenu moyen des femmes est moindre de $31 \%$ (dans le cirque) à $58 \%$ (dans les arts de la rue) (graphique 2). 
Graphique 2 - Revenus moyens perçus par les femmes et les hommes pour la création audiovisuelle et de spectacle vivant, 2011

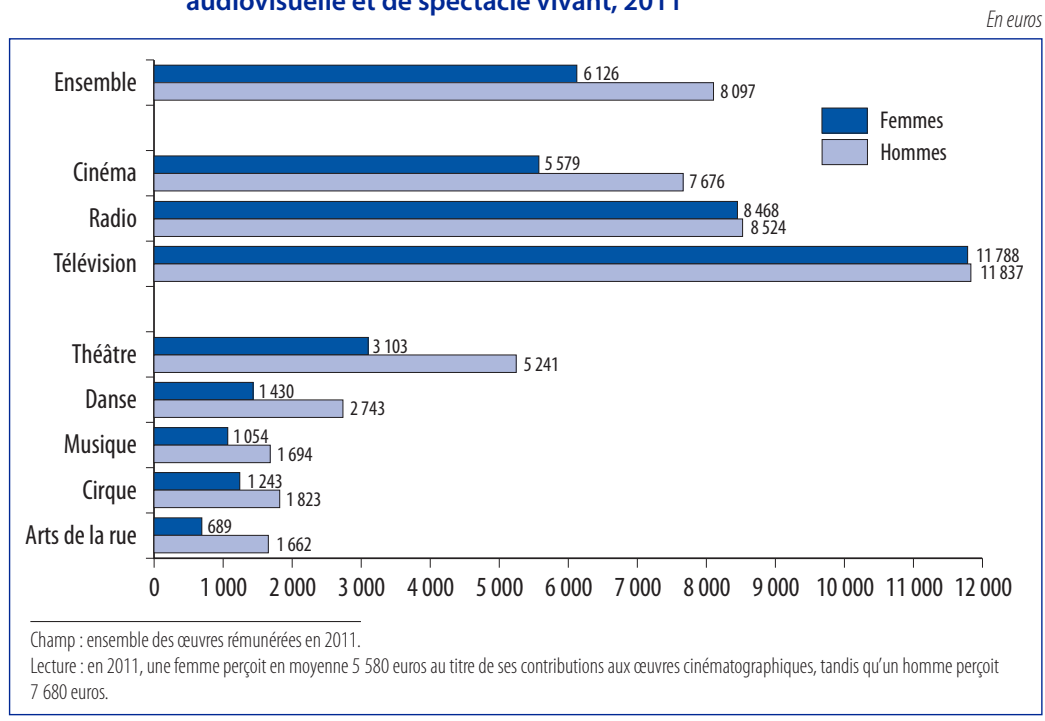

Source : SACD/DEPS, Ministère de la Culture et de Communication, 2014

\section{Moins de créations féminines que masculines, en particulier dans le cinéma, le théâtre et les arts de la rue}

Un premier élément d'explication de ces écarts a trait à la moindre participation des femmes à la création audiovisuelle et de spectacle vivant. Les femmes déclarent en effet $27 \%$ des contributions aux œuvres rémunérées en 2011, qu'elles soient auteur unique de l'œuvre ou en partagent la création avec d'autres co-auteurs (voir glossaire) : en moyenne, en 2011, une femme est rémunérée au titre de 14,3 contributions, contre 16,1 contributions en moyenne pour un homme (tableau 1).

L'écart est particulièrement marqué dans le théâtre - où la population féminine représente $31 \%$ des auteurs mais ne contribue qu'à $27 \%$ des créations - ainsi que le cinéma et les arts de la rue, où les femmes représentent un quart des auteurs et sont à l'origine de $21 \%$ des contributions. Dans ces disciplines, en effet, le nombre moyen de contributions rémunérées déclarées par les femmes est inférieur de près de $20 \%$ à celui de leurs homologues masculins. Ailleurs, comme dans la radio, la musique et le cirque, l'écart est de l'ordre de $10 \%$, et pratiquement inexistant dans la danse et la télévision.

\section{Dans la plupart des disciplines, la création d'une œuvre repose sur l'écriture de texte}

Dans le registre de la SACD, les auteurs sont identifiés selon leur fonction dans la création. Ils peuvent être auteurs de texte dans les différentes disciplines audiovisuelles - scénaristes, dialoguistes, etc. - et du spectacle vivant, réalisateurs pour le cinéma ou la télévision, metteurs en scène pour le théâtre, chorégraphes ou compositeurs pour le spectacle vivant.

Dans la plupart des disciplines, l'écriture de texte est la fonction la plus couramment exercée. Elle est l'unique fonction à l'œuvre dans la création radiophonique et domine largement la création pour le théâtre ( $92 \%$ des contributions à cette discipline - les 
Tableau 1 - Contributions moyennes et totales, masculines et féminines, rémunérées dans l'audiovisuel et le spectacle vivant, 2011

\begin{tabular}{|c|c|c|c|c|c|c|}
\hline & \multicolumn{2}{|c|}{ Nombre total de contributions } & \multicolumn{4}{|c|}{ Nombre moyen de contributions... } \\
\hline & Ensemble & $\begin{array}{c}\text { dont } \% \\
\text { déclarées par } \\
\text { des femmes }\end{array}$ & Ensemble & $\begin{array}{c}\text { par } \\
\text { femme }\end{array}$ & $\begin{array}{c}\text { par } \\
\text { homme }\end{array}$ & $\begin{array}{c}\text { Écart femme/ } \\
\text { homme } \\
\text { (en \%) }\end{array}$ \\
\hline Audiovisuel & 254481 & 27 & 29,6 & 29,3 & 29,7 & -1 \\
\hline Cinéma & 6226 & 21 & 1,8 & 1,5 & 1,8 & -18 \\
\hline Radio & 10530 & 32 & 26,5 & 24,8 & 27,3 & -9 \\
\hline Télévision & 237725 & 27 & 41,5 & 41,2 & 41,6 & -1 \\
\hline Spectacle vivant & 25436 & 27 & 2,4 & 2,1 & 2,6 & -17 \\
\hline Théâtre & 16248 & 27 & 2,5 & 2,2 & 2,7 & -19 \\
\hline Danse & 3441 & 34 & 2,1 & 2,1 & 2,1 & 0 \\
\hline Musique & 3391 & 21 & 1,5 & 1,4 & 1,6 & -12 \\
\hline Cirque & 953 & 26 & 1,4 & 1,3 & 1,4 & -9 \\
\hline Arts de la rue & 1403 & 21 & 1,7 & 1,4 & 1,8 & -21 \\
\hline Ensemble & 279917 & 27 & 15,5 & 14,3 & 16,1 & -11 \\
\hline
\end{tabular}

Source: SACD/DEPS, Ministère de la Culture et de Communication, 2014

$8 \%$ restants se partageant entre mise en scène et composition musicale). L'écriture de texte est également majoritaire dans le cirque (75\% des contributions), les arts de la rue (70\%), la télévision (69\%) et le cinéma (60\%) (tableau 2).

Tableau 2 - Part des différentes fonctions exercées dans la création d'une œuvre rémunérée, 2011

\begin{tabular}{|c|c|c|c|c|c|c|c|}
\hline & Réalisateur & $\begin{array}{l}\text { Auteur } \\
\text { de texte }\end{array}$ & $\begin{array}{l}\text { Autre } \\
\text { fonction } \\
\text { audiovisuelle }\end{array}$ & $\begin{array}{l}\text { Metteur } \\
\text { en scène }\end{array}$ & $\begin{array}{l}\text { Choré- } \\
\text { graphe }\end{array}$ & $\begin{array}{l}\text { Compo- } \\
\text { siteur }\end{array}$ & Ensemble \\
\hline Audiovisuel & 24 & 70 & 6 & I & 1 & I & 91 \\
\hline Cinéma & 40 & 60 & 0 & l & l & l & 2 \\
\hline Radio & l & 100 & 0 & I & l & l & 4 \\
\hline Télévision & 24 & 69 & 6 & I & I & I & 85 \\
\hline Spectacle vivant & l & 72 & l & 3 & 10 & 15 & 9 \\
\hline Théâtre & l & 92 & l & 5 & 0 & 3 & 6 \\
\hline Danse & l & 19 & I & l & 58 & 23 & 1 \\
\hline Musique & l & 42 & I & l & 2 & 56 & 1 \\
\hline Cirque & l & 75 & I & l & 7 & 18 & 0 \\
\hline Arts de la rue & I & 70 & I & I & 7 & 23 & 1 \\
\hline Ensemble & 22 & 70 & 6 & 0 & 1 & 1 & 100 \\
\hline \multicolumn{8}{|c|}{$\begin{array}{l}\text { Champ : ensemble des œeuvres rémunérées en } 2011 . \\
\text { Lecture: dans l'ensemble des contributions rémunérées en } 2011,2 \% \text { sont cinématographiques. Parmi celles-i, } 40 \text { \% relèvent de la réalisation et } 60 \% \text { de } \\
\text { l'écriture (scénario, dialogues, etc.). La fonction de réalisateur concentre } 22 \% \text { de l'ensemble des contributions rémunérées des deux répertoires, audiovisuel et } \\
\text { spectacle vivant. }\end{array}$} \\
\hline
\end{tabular}


La création musicale repose d'abord sur la composition (56\% des contributions aux œuvres musicales) et la danse sur la chorégraphie (58\% des contributions à cette discipline). Compositeurs et chorégraphes interviennent également dans le cirque et les arts de la rue (autour de $20 \%$ pour les premiers, moins de $10 \%$ pour les seconds). La composition musicale est, de plus, une fonction couramment exercée pour créer une œuvre chorégraphique ( $23 \%$ des contributions).

La réalisation représente $40 \%$ des contributions aux œuvres cinématographiques, mais une part nettement moindre dans la création télévisuelle $(24 \%)$, davantage investie par l'écriture de texte, où il est très fréquent que plusieurs co-auteurs participent à l'écriture d'une même œuvre télévisuelle.

\section{Les femmes sont d'abord chorégraphes ou auteurs de l'écrit}

Une contribution sur trois en qualité d'auteur de texte est féminine, plus souvent dans la radio, la télévision, le théâtre, la danse, la musique et le cirque (autour de $30 \%$ des contributions en tant qu'auteur de texte) que dans le cinéma et les arts de la rue (tableau 3).

La chorégraphie est la fonction traditionnellement la plus féminisée, bien que les femmes y soient, là aussi, minoritaires : $45 \%$ des contributions au titre de la fonction chorégraphique sont féminines, toutes disciplines du spectacle vivant confondues.

\section{Les réalisatrices et les compositrices sont rares}

La réalisation et la composition musicale sont nettement masculinisées. Les femmes ne sont à l'origine que de $19 \%$ des contributions en tant que réalisateur pour les œuvres de cinéma, et seulement de $7 \%$ pour les œuvres télévisuelles. Elles déclarent autour de $10 \%$ des contributions au titre de la composition musicale, que ce soit pour les œuvres musicales, chorégraphiques, circassiennes ou d'arts de la rue (tableau 3).

Tableau 3 - Fonctions exercées par les femmes dans la création d'œuvres audiovisuelles et de spectacle vivant, 2011

\begin{tabular}{|c|c|c|c|c|c|c|c|}
\hline & Réalisateur & $\begin{array}{l}\text { Auteur } \\
\text { de texte }\end{array}$ & $\begin{array}{l}\text { Autre } \\
\text { fonction } \\
\text { audiovisuelle }\end{array}$ & $\begin{array}{l}\text { Metteur } \\
\text { en } \\
\text { scène }\end{array}$ & $\begin{array}{l}\text { Choré- } \\
\text { graphe }\end{array}$ & $\begin{array}{l}\text { Compo- } \\
\text { siteur }\end{array}$ & Ensemble \\
\hline Audiovisuel & 8 & 32 & 12 & I & I & I & 27 \\
\hline Cinéma & 19 & 22 & I & I & I & I & 21 \\
\hline Radio & I & 32 & I & I & I & I & 32 \\
\hline Télévision & 7 & 33 & 12 & l & I & I & 27 \\
\hline Spectacle vivant & I & 27 & I & 27 & 45 & 12 & 27 \\
\hline Théâtre & I & 27 & I & 27 & l & 17 & 27 \\
\hline Danse & l & 32 & l & l & 44 & 9 & 34 \\
\hline Musique & I & 30 & I & I & 55 & 13 & 21 \\
\hline Cirque & I & 28 & I & 1 & 40 & 11 & 26 \\
\hline Arts de la rue & I & 23 & I & I & 42 & 5 & 21 \\
\hline Ensemble & 8 & 32 & 12 & 27 & 45 & 12 & 27 \\
\hline \multicolumn{8}{|c|}{$\begin{array}{l}\text { Champ : ensemble des œuvres rémunérées en } 2011 . \\
\text { Lecture : } 19 \% \text { des contributions aux ceuvres de cinéma en tant que réalisateur sont déclarées par des femmes ; } 8 \% \text { de l'ensemble des contributions en tant } \\
\text { que réalisateur, toutes disciplines confondues, sont déclarées par des femmes ; } 21 \% \text { de l'ensemble des contributions aux œuuvres de cinéma sont déellarées } \\
\text { par des femmes. }\end{array}$} \\
\hline
\end{tabular}




\section{Pour la radio et la danse, les femmes créent plus souvent seules}

La plupart des œuvres chorégraphiques, cinématographiques, musicales et, particulièrement, les œuvres théâtrales et radiophoniques sont créées ou composées par un auteur unique. À l'inverse, dans les arts de la rue, le cirque et, plus encore, la télévision, elles sont l'œuvre de la collaboration de plusieurs auteurs, parfois nombreux (tableau 4). Les œuvres à auteurs multiples représentent ainsi $83 \%$ de l'ensemble des œuvres télévisuelles et sont créées par plus de quatre co-auteurs en moyenne. Cette multiplicité de co-auteurs est caractéristique notamment des épisodes de séries télévisées, créés au sein d'ateliers d'écriture, qui impliquent la mobilisation simultanée d'un grand nombre de contributeurs.

Pour autant, la répartition entre femmes et hommes n'est pas la même selon que les œuvres sont créées par un auteur unique ou par plusieurs co-auteurs. Ainsi, dans la danse et la radio, les femmes représentent $41 \%$ des auteurs uniques d'œuvres chorégraphiques et radiophoniques. Mais lorsque ces œuvres sont le fruit de plusieurs co-auteurs, les femmes sont nettement moins représentées (31\% pour la danse et $18 \%$ pour la radio) (tableau 4 ).

Tableau 4 - Part des femmes dans les contributions aux œuvres à auteur unique et à auteurs multiples rémunérées, 2011

\begin{tabular}{|c|c|c|c|c|c|c|c|c|}
\hline & \multirow{2}{*}{$\begin{array}{c}\text { Nombre } \\
\text { total } \\
\text { d'œuvres }\end{array}$} & \multicolumn{3}{|c|}{ à auteur unique } & \multicolumn{4}{|c|}{ à co-auteurs multiples } \\
\hline & & $\%$ & $\begin{array}{c}\text { Nombre } \\
\text { de } \\
\text { contributions }\end{array}$ & $\begin{array}{l}\text { dont } \% \text { de } \\
\text { contributions } \\
\text { féminines }\end{array}$ & $\%$ & $\begin{array}{c}\text { Nombre } \\
\text { de } \\
\text { contributions }\end{array}$ & $\begin{array}{l}\text { Nombre } \\
\text { moyen de } \\
\text { co-auteurs } \\
\text { par œuvre }\end{array}$ & $\begin{array}{l}\text { dont } \% \text { de } \\
\text { contributions } \\
\text { féminines }\end{array}$ \\
\hline Audiovisuel & 74469 & 26 & 19007 & 25 & 74 & 235474 & 4,2 & 27 \\
\hline Cinéma & 3689 & 55 & 2017 & 21 & 45 & 4209 & 2,5 & 21 \\
\hline Radio & 7860 & 81 & 6340 & 41 & 19 & 4190 & 2,8 & 18 \\
\hline Télévision & 62920 & 17 & 10650 & 16 & 83 & 227075 & 4,3 & 27 \\
\hline Spectacle vivant & 16924 & 68 & 11567 & 25 & 32 & 13869 & 2,6 & 28 \\
\hline Théâtre & 11947 & 75 & 8967 & 25 & 25 & 7281 & 2,4 & 29 \\
\hline Danse & 1974 & 51 & 1005 & 41 & 49 & 2436 & 2,5 & 31 \\
\hline Musique & 2030 & 61 & 1247 & 14 & 39 & 2144 & 2,7 & 25 \\
\hline Cirque & 338 & 23 & 78 & 28 & 77 & 875 & 3,4 & 26 \\
\hline Arts de la rue & 635 & 43 & 270 & 16 & 57 & 1133 & 3,1 & 22 \\
\hline Ensemble & 91393 & 33 & 30574 & 25 & 67 & 249343 & 4,1 & 27 \\
\hline
\end{tabular}

Source: SACD/DEPS, Ministère de la Culture et de Communication, 2014

\section{Pour la télévision, la musique et les arts de la rue, elles interviennent davantage en tant que co-auteurs}

La contribution féminine à la musique concerne un quart des œuvres à auteurs multiples, contre $14 \%$ des œuvres à auteur unique. Les proportions sont assez semblables pour les arts de la rue ( $22 \%$ des contributions aux œuvres à auteurs multiples sont féminines, soit 6 points de plus que pour les œuvres créées en solo) et la télévision ( $27 \%$ de contributions aux œuvres à auteurs multiples sont féminines et $16 \%$ des créations en solo). 


\section{Au moment de la déclaration de l'œuvre, pour une fonction donnée, la répartition des droits est égalitaire entre co-auteurs, qu'ils soient hommes ou femmes}

Sur le bulletin de déclaration de l'œuvre (voir glossaire), la répartition entre coauteurs s'opère de façon différente dans le cinéma et la télévision d'une part, dans la radio et le spectacle vivant d'autre part.

Pour une œuvre de cinéma ou de télévision, la définition des parts (dites « parts au bulletin ») entre réalisateurs et auteurs de texte est le fruit d'un usage établi. Pour la radio et le spectacle vivant, elle s'effectue de gré à gré entre co-auteurs. Dans l'un et l'autre cas, la répartition des parts entre co-auteurs est égalitaire entre femmes et hommes, les unes ayant une part au bulletin, en moyenne, comparable à celles des autres, quelle que soit la discipline artistique et pour une fonction donnée. Par exemple, un homme réalisateur et une femme scénariste déclarant un film de cinéma n'auront pas la même part au bulletin ; suivant l'usage établi, $40 \%$ seront attribués au réalisateur et $60 \%$ à la scénariste. Si trois co-auteurs déclarent le film - un réalisateur, une réalisatrice et une scénariste - alors l'homme et la femme réalisateurs se répartiront $40 \%$ à parts en moyenne égales, soit $20 \%$ chacun, et $60 \%$ reviendront à la scénariste.

Toutefois, si les parts attribuées au moment de la déclaration de l'œuvre sont similaires pour les hommes et pour les femmes, il n'en va pas toujours de même des montants effectivement perçus une fois l'œuvre diffusée ou représentée.

\section{La rémunération d'une femme au titre de la réalisation ou de l'écriture de texte est légèrement inférieure à celle d'un homme pour une œuvre de cinéma...}

Lorsque les femmes participent à la création d'une œuvre de cinéma, la rémunération de leurs contributions en tant que réalisatrices ou auteurs de texte est légèrement inférieure à celles des hommes. Une contribution en tant que réalisateur est rémunérée en moyenne 2385 euros pour une femme et 2770 euros pour un homme ; l'écart est moindre pour un auteur de texte : 2400 euros en moyenne d'une part, 2555 euros de l'autre (tableau 5). Ainsi, à fonctions identiques, les femmes perçoivent des droits légèrement inférieurs à ceux des hommes alors que le partage des droits entre co-auteurs, au moment de la déclaration de l'œuvre de cinéma, induit des parts comparables entre hommes et femmes.

On peut faire l'hypothèse que les femmes cinéastes ne participent pas au même type d'œuvres que leurs homologues masculins (courts et longs métrages n'offrent pas les mêmes rémunérations), que ces œuvres ne bénéficient pas de la même diffusion (rediffusions sur des chaînes de télévision nationales, de la TNT ou privées, etc.), ni des mêmes plages horaires, ni de la même fréquence.

\section{... mais de même niveau pour une œuvre télévisuelle}

En 2011, les droits moyens perçus par les femmes et les hommes pour la contribution à une œuvre de télévision sont en revanche comparables : environ 300 euros pour l'écriture de texte, 200 euros pour une réalisation. Les droits moyens associés à l'exercice de ces fonctions sont nettement moins importants à la télévision qu'au cinéma car les droits sont partagés entre un plus grand nombre de co-auteurs et concernent des œuvres de formats souvent beaucoup plus courts, moins rémunérées.

\section{Pour une œuvre radiophonique, les femmes perçoivent des droits en moyenne plus élevés que les hommes}

La création d'une œuvre radiophonique repose presque exclusivement sur la seule fonction d'auteur de texte. Les droits que les femmes retirent, en moyenne, 
Tableau 5 - Droits moyens perçus par les femmes et par les hommes pour la contribution à une œuvre au titre d'une fonction donnée, 2011

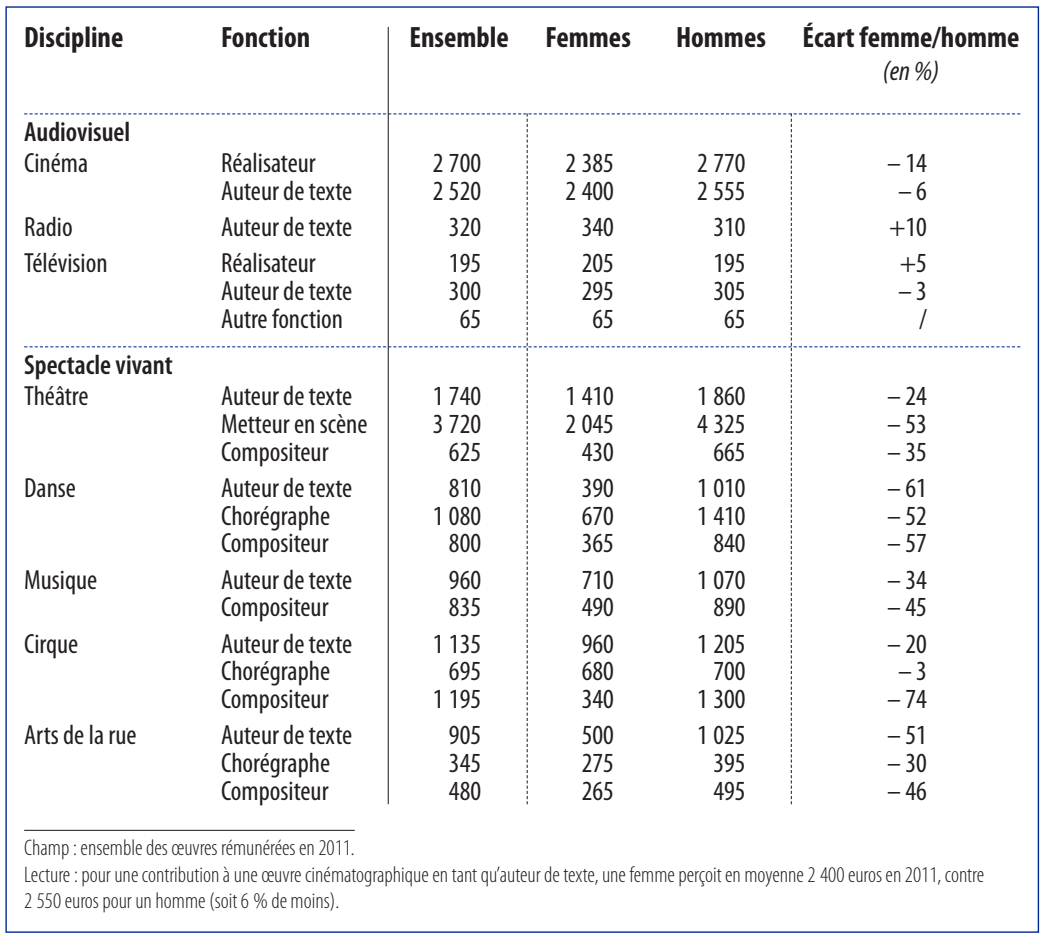

Source : SACD/DEPS, Ministère de la Culture et de Communication, 2014

d'une contribution à une œuvre de radio dépassent légèrement ceux des hommes: 340 euros contre 310 euros. On explique cet écart par le fait que les hommes de radio sont plus souvent co-auteurs que les femmes et donc plus souvent amenés à partager les droits d'une œuvre donnée: en effet, on l'a vu, si $59 \%$ des œuvres de radio à auteur unique sont déclarées par des hommes, $82 \%$ des contributions aux œuvres à auteurs multiples sont masculines dans cette discipline (tableau 4).

\section{Dans le spectacle vivant, les droits associés aux contributions féminines sont inférieurs à ceux des hommes, quelles que soient la discipline et la fonction}

Dans le spectacle vivant aussi, quelle que soit la fonction exercée pour créer une œuvre, on relève d'importants écarts de revenus entre hommes et femmes, alors que les parts au bulletin des unes et des autres - c'est-à-dire le partage après création de l'œuvre mais avant sa diffusion - sont comparables pour l'exercice d'une fonction donnée.

Par exemple, pour une œuvre théâtrale, un homme auteur de texte est rémunéré 1860 euros en moyenne en 2011 ; pour l'exercice de la même fonction, une femme perçoit en moyenne 1410 euros, soit $24 \%$ de moins. Dans les autres disciplines du spectacle vivant, les droits moyens perçus par une femme pour son intervention en tant que chorégraphe ou compositrice sont très inférieurs à ceux des hommes (jusqu'à quatre fois moins pour une composition musicale destinée à une œuvre circassienne). 
Il est vraisemblable que ces écarts reposent sur la nature différente des œuvres proposées par les unes et les autres, leur succès, la capacité d'accueil des lieux où ces œuvres sont représentées et peut-être une moindre programmation des œuvres féminines. Ainsi, les femmes participent moins que les hommes à la création des œuvres audiovisuelles ou de spectacle vivant. Lorsqu'elles y contribuent, c'est d'abord pour la radio et la danse, moins pour le cinéma, la musique et les arts de la rue que pour le cirque, le théâtre, la télévision. Elles sont plus souvent auteurs de texte ou chorégraphes que réalisatrices ou compositrices.

Lorsqu'elles participent à l'élaboration d'œuvres radiophoniques ou télévisuelles, elles sont rétribuées autant voire un peu plus que les hommes, tant en termes de revenus perçus en 2011 que de part des droits d'auteur prévue au moment de la déclaration de l'œuvre à la SACD. Sachant qu'elles sont rémunérées pour un nombre de contributions légèrement inférieur à celui des hommes dans ces disciplines (tableau 1), in fine, les revenus totaux qu'elles tirent de leurs créations pour la radio et la télévision sont en moyenne équivalents à ceux des hommes (graphique 2).

À l'inverse, leurs contributions aux œuvres de cinéma et, plus encore, de spectacle vivant, quelle que soit la fonction au titre de laquelle elles interviennent, sont moins rémunérées que celles des hommes alors que les parts qui leur sont destinées sont comparables, ce qui tend à confirmer l'hypothèse selon laquelle les femmes de cinéma et du spectacle vivant ne participent pas au même type d'œuvres que leurs homologues masculins, que ces œuvres ne sont pas représentées dans les mêmes lieux ou diffusées sur les mêmes chaînes de télévision, ni aux mêmes horaires, ni à la même fréquence.

\section{POUR EN SAVOIR PLUS}

- Gouron Marie, Diversité des carrières d'auteur de l'audiovisuel et du spectacle vivant. Les auteurs de la SACD, 1997-2008, Ministère de la Culture et de la Communication, DEPS, coll. « Culture chiffres », 2011-2, avril 2011 - Ministère de la Culture et de la Communication (2014), « Rapport de l'Observatoire de l'égalité femmes/hommes dans la culture et la communication », mars 2014, disponible sur www.culturecommunication.gouv.fr, rubrique «Égalité entre femmes et hommes » 


\section{Encadré 1}

\section{La SACD : rôle et fonctionnement}

\section{Une société de gestion des droits d'auteur}

Intermédiaires entre les auteurs et les producteurs d'œuvres soumis au droit d'auteur, les sociétés de gestion des droits d'auteur, ou sociétés de perception et de répartition des droits (SPRD), exercent la gestion collective des droits des auteurs; elles ont un statut de droit privé mais exercent néanmoins une mission reconnue d'utilité publique sous la tutelle du ministère de la Culture et de la Communication.

La SACD (Société des auteurs et compositeurs dramatiques), créée en 1777, est l'une des 22 SPRD reconnues en France, au même titre que la Sacem (Société des auteurs, compositeurs et éditeurs de musique) et que la Scam (Société civile des auteurs multimédias) par exemple.

\section{Les répertoires : I'audiovisuel et le spectacle vivant}

Pour le spectacle vivant, la SACD est compétente pour les œuvres de théâtre, les œuvres dramatico-musicales (les opéras, les comédies musicales, les œuvres de théâtre musical, les musiques de scène - la musique enregistrée relève du champ de compétence de la Sacem), les chorégraphies, les sketches, les one-man-show, les mises en scène, les œuvres de cirque, les œuvres d'arts de la rue, les œuvres de mimes, de marionnettes et les sons et lumières.

Dans l'audiovisuel, elle gère les droits issus de la diffusion télévisuelle des œuvres de cinéma (courts et longs métrages) et de télévision (téléfilms, séries télévisées, feuilletons, œuvres d'animation) ainsi que de la diffusion des fictions radiophoniques. Les œuvres documentaires, quant à elles, relèvent de la Scam.

\section{L'adhésion à la SACD}

Une personne peut adhérer à la SACD si elle est dans l'une des situations suivantes:

- elle est l'auteur d'une œuvre déjà créée ou en cours de création, relevant du répertoire de la SACD ;

- elle est l'héritier d'un auteur décédé, non-membre de la SACD de son vivant, dont l'œuvre va être représentée ou diffusée;

- elle est auteur en stage de fin d'études ou diplômée d'une école de cinéma, d'un conservatoire d'écriture ou d'un établissement similaire reconnu par la SACD ;

- elle est auteur parrainé par deux sociétaires de la SACD. 


\section{Encadré 2}

\section{Les disciplines artistiques : œuvres, droits versés en 2011, auteurs}

En 2011, parmi les œuvres donnant lieu au versement de droits d'auteur, une nette majorité (74 470) relève de l'audiovisuel, et en particulier de la télévision (62 920), en raison du format très court d'un grand nombre de programmes télévisuels (par exemple, les « pastilles», de moins de 2 minutes, intercalées entre deux émissions) et du poids des séries télévisées. Les œuvres de radio et de cinéma sont significativement moins nombreuses : 7860 œuvres concernent la radio et 3690 concernent les œuvres cinématographiques pour leurs diffusions télévisuelles.

Dans le spectacle vivant, 16930 œuvres sont rémunérées en 2011, dont une majorité d'œuvres théâtrales (11 950). La danse et la musique rassemblent chacune autour de 2000 œuvres, tandis que les arts de la rue et le cirque réunis en rassemblent près de 1000 (respectivement 640 et 340).

En 2011, près de 100 millions d'euros de droits nets ont été répartis entre les auteurs au titre de la diffusion d'œuvres audiovisuelles, en particulier télévisuelles (67,7 millions d'euros) et cinématographiques (25,4 millions d'euros) et, plus marginalement, radiophoniques (3,4 millions d'euros). Près de 40 millions d'euros de droits nets sont partagés entre les auteurs du spectacle vivant, avec le théâtre au premier chef (29,4 millions d'euros) et, dans une moindre mesure, la danse et la musique (un peu moins de 4 millions d'euros chacune), puis le cirque et les arts de la rue (un peu plus d'un million d'euros chacun en 2011).

Ces droits sont versés à 18000 auteurs actifs (voir glossaire). La plupart d'entre eux (95\%) sont vivants ; lorsqu'ils sont décédés, les droits d'auteur générés par la diffusion ou la représentation de leurs œuvres sont versés à leurs ayants droit, jusqu'à ce que l'œuvre tombe dans le domaine public. Parmi les auteurs actifs, 48 \% interviennent dans l'audiovisuel, principalement dans la télévision (5 730 auteurs), puis le cinéma (3 550 auteurs) et de façon beaucoup plus marginale à la radio (400 auteurs). Le spectacle vivant rassemble $58 \%$ des auteurs actifs de la SACD, dont une majorité dans le théâtre (6 420 auteurs); 2240 auteurs sont rémunérés en 2011 au titre d'œuvres musicales, 1660 pour la danse, 820 pour les arts de la rue et près de 700 pour le cirque.

Certains auteurs sont actifs dans plusieurs disciplines d'un même répertoire, ou même sur les deux répertoires : 500 auteurs sont rémunérés en 2011 à la fois pour des œuvres audiovisuelles et pour des contributions au spectacle vivant.

En 2011, les auteurs ont en moyenne 48 ans. Les femmes sont plus jeunes que les hommes à contribuer aux différentes disciplines : 47 ans en moyenne, contre 49 ans pour leurs homologues masculins, du fait de la féminisation récente de la création dans l'audiovisuel et le spectacle vivant ${ }^{1}$.

Certaines disciplines sont plus jeunes que d'autres : le cirque est la discipline de loin la plus jeune avec une moyenne à 41 ans, tandis que les auteurs ont en moyenne 45 ans dans le cinéma, la danse et les arts de la rue, et 50 ans en moyenne dans la radio, la télévision et le théâtre.

L'écart d'environ deux ans, au profit des femmes, s'observe dans presque toutes les disciplines, à l'exception notable de la danse où les femmes sont en moyenne plus âgées (46 ans) que les hommes (44 ans), en raison de la longue tradition d'ouverture de la danse aux femmes.

1. voir GouYon M., « Diversité des carrières d'auteur de l'audiovisuel et du spectacle vivant. Les auteurs de la SACD, 1997-2008», coll. « Culture Chiffres », 2011-2. 
Graphique A - Répartition par discipline des œuvres, droits et auteurs rémunérés dans l'audiovisuel et le spectacle vivant, 2011

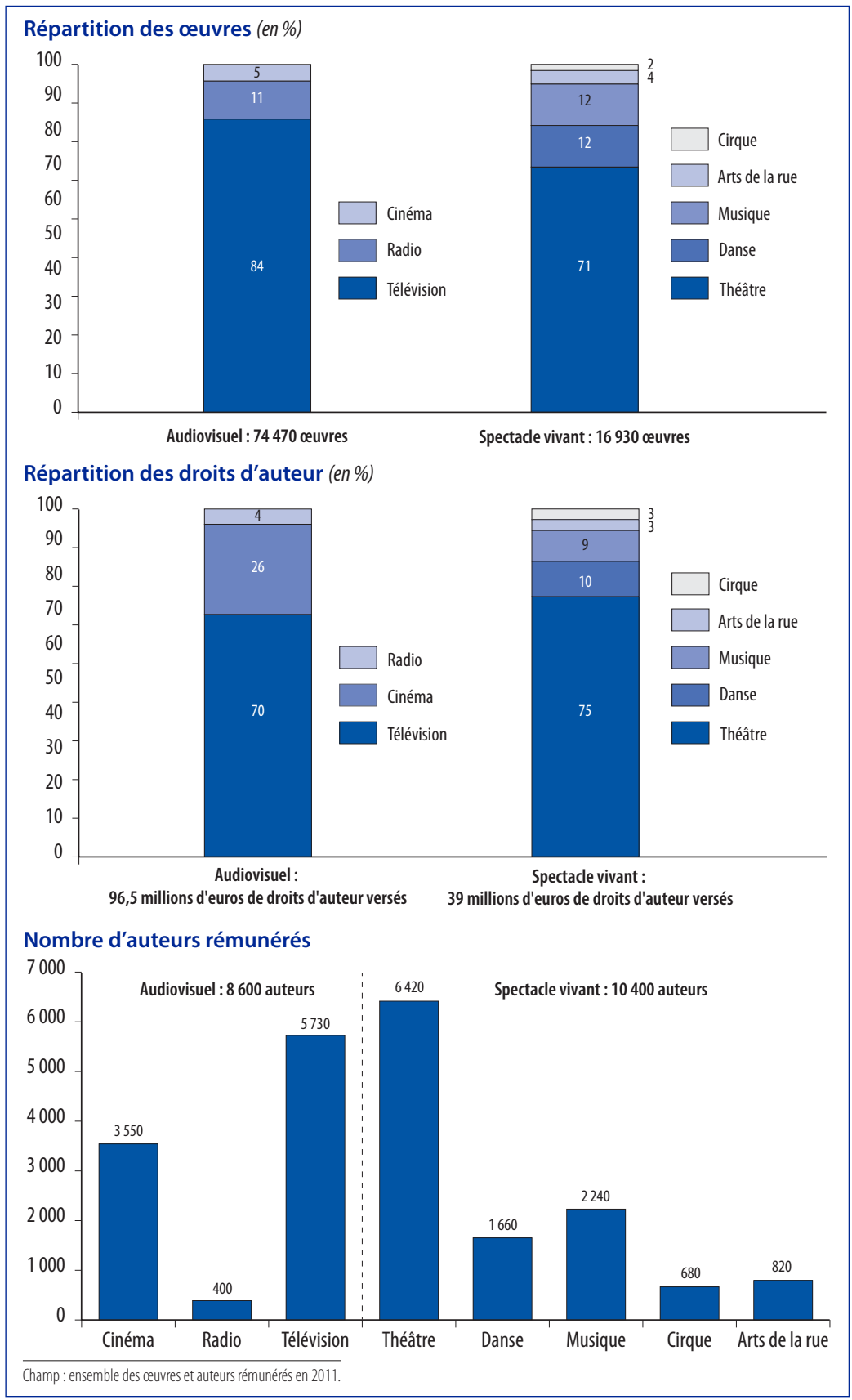




\section{Encadré 3}

\section{Méthodologie}

L'analyse porte sur les œuvres qui donnent lieu à répartition, en 2011 par la SACD, de droits d'auteur en gestion collective. Par conséquent, le comportement de création étudié ici ne porte pas sur l'ensemble de la carrière d'un auteur, mais sur la partie de son œuvre qui a été récemment diffusée ou représentée. Les auteurs étudiés sont ainsi considérés comme actifs: leurs contributions sont diffusées ou représentées, générant ainsi des droits d'auteur en 2011

On dispose notamment, pour chaque œuvre donnant lieu à rémunération en 2011 : - de ses caractéristiques : répertoire, discipline (par exemple la télévision pour une œuvre audiovisuelle), genre artistique (par exemple une fiction télévisuelle).

Pour chaque auteur ayant participé à la création de cette œuvre :

- de caractéristiques sociodémographiques telles que sexe, année de naissance et le cas échéant de décès ;

- de la ou des fonction(s) qu'il a exercée(s) (voir glossaire).

Enfin, pour chaque auteur et chaque fonction qu'il a exercée pour participer à la création de l'œuvre:

- de la part au bulletin (en \%) (voir glossaire);

- du montant net de droits d'auteur perçus en 2011 (en euros).

\section{Glossaire}

Arche narrative : pour une œuvre télévisuelle, document écrit présentant de façon succincte le parcours des personnages récurrents d'une série et l'évolution de leurs relations sur tout ou partie d'une saison.

Argument : trame narrative d'une œuvre chorégraphique.

Auteur actif : tout auteur dont les contributions sont diffusées ou représentées, générant ainsi des droits d'auteur en 2011.

Bible littéraire : document de référence original et fondateur d'une série télévisée, déterminant et décrivant les éléments nécessaires à l'écriture, par des auteurs différents, des épisodes d'une œuvre télévisuelle. Elle donne aux auteurs qui collaborent ou collaboreront à l'œuvre les clés de son fonctionnement et de sa cohérence.

Bulletin de déclaration : document contractuel signé par l'auteur ou les différents co-auteurs de l'œuvre, remis à la SACD pour visa au moment de la déclaration. Ce bulletin décline l'identité civile de l'œuvre (titre, genre, durée, lieu et date de création) et constitue le contrat passé entre les co-auteurs éventuels pour le partage de leurs droits (parts au bulletin) en fonction de l'apport de chacun.

Contribution : participation d'un auteur à une œuvre donnée.

Création : genèse d'une œuvre audiovisuelle ou de spectacle vivant. Pour ce dernier répertoire, le terme ne couvre pas la «création » au sens de «première représentation ».

Fonction : activité(s) exercée(s) par un auteur ou co-auteur pour contribuer à la création d'une œuvre.

Dans l'audiovisuel, les auteurs peuvent être :

- réalisateurs (pour le cinéma ou la télévision); 
- auteurs de texte : scénaristes, dialoguistes, auteurs littéraires, dramatiques, traducteurs, adaptateurs de texte, auteurs d'une œuvre préexistante. Également, pour les œuvres télévisuelles, auteurs du synopsis, de l'arche narrative, de la bible littéraire ;

- créateurs de marionnettes, de personnages, graphistes, traducteurs, compositeurs, chorégraphes, etc.

Dans le spectacle vivant, ils peuvent être:

- auteurs de texte : auteurs dramatiques, auteurs littéraires ou poètes, auteurs du montage de texte, adaptateurs de livret ou librettistes, traducteurs, auteurs ou adaptateurs de l'argument, auteur d'une œuvre préexistante ;

- chorégraphes (ou adaptateurs de chorégraphie) ;

- compositeurs (ou adaptateurs de la musique);

- metteurs en scène, pour une œuvre théâtrale. La déclaration de la mise en scène n'est pas obligatoire à la SACD.

Musique de scène : musique originale accompagnant la représentation d'un ouvrage dramatique appartenant au répertoire de la SACD. Si la musique est préexistante, elle relève du répertoire de la Sacem, ou encore du domaine public.

Part au bulletin ou part après visa : part des droits attribuée, sur le bulletin de déclaration, à chaque contributeur au titre de sa fonction, ou de chacune de ses fonctions, qu'il exerce dans la création de l'œuvre. Il s'agit d'un pourcentage des droits d'auteur sur une base de $100 \%$ qui sera appliqué à la rémunération de toutes les diffusions ou représentations de l'œuvre. Cette part résulte du partage des droits entre co-auteurs et figure sur le bulletin de déclaration.

- Pour les œuvres de spectacle vivant et de radio, les parts au bulletin sont fixées de gré à gré par les différents co-auteurs eux-mêmes si plusieurs auteurs sont à l'origine de l'œuvre.

- Pour les œuvres cinématographiques et télévisuelles, des règles spécifiques adoptées par le conseil d'administration sont appliquées au partage des droits. Ainsi, pour une œuvre de cinéma, la part attribuée à la réalisation est, systématiquement, de $40 \%$. Les $60 \%$ restants sont affectés à (aux) auteur(s) du texte.

Au moment de la déclaration d'une œuvre de télévision, deux bulletins spécifiques sont complétés, au titre de la réalisation d'une part, des autres fonctions d'autre part. Les parts mentionnées sur chacun de ces deux bulletins s'appuient sur une base de $100 \%$. Une clé de répartition complémentaire est appliquée lors de la répartition des droits. Ainsi, les parts des droits attribuées à la réalisation d'un côté et aux autres fonctions de l'autre sont ramenées respectivement à $10 \%$ et 90 \% pour une première diffusion sur les chaînes nationales historiques (TF1, France 2, France 3, France 5, M6, Arte, Canal plus) puis à $20 \%$ et $80 \%$ pour toutes les autres diffusions et tous les autres diffuseurs.

Pour le cinéma comme pour la télévision, en cas de coréalisation ou de co-auteurs pour l'écriture de texte ou l'exercice d'autres fonctions, les droits se partagent de gré à gré.

Synopsis: résumé du scénario d'un film, décrivant les grandes lignes de l'histoire, esquissant les principaux personnages et leur évolution.

Pour davantage de définitions, on se reportera au glossaire mis en ligne par la SACD à l'adresse suivante : http://www.sacd.fr/Glossaire.265.0.html 


\title{
Abstract
}

\section{Women in audio-visual and live show creation SACD (Society of Authors and Dramatists) authors receiving royalties in 2011}

\begin{abstract}
In 2011, women represented 29\% of SACD authors and received 24\% of the royalties paid. As a result, in 2011, a woman received an average income 24\% less than that for a man.

The differences in royalties between men and women can be explained in several ways: women participate in fewer works than men, in particular in the cinema, theatre and street arts. Neither do they perform the same functions, and so are more often text authors or choreographers than directors or composers.

For the same discipline and function the differences in remuneration are practically non-existent in radio and television, but live on in cinema and live shows: these persistent differences can be linked to other factors, such as, for example, lower audience numbers or programming of works, in different performance venues or on different distribution channels and at less favourable times.
\end{abstract}

\section{À lire aussi}

\section{CC-2011-2}

\section{Diversité des carrières d'auteur de l'audiovisuel et du spectacle vivant Les auteurs de la SACD, 1997-2008}

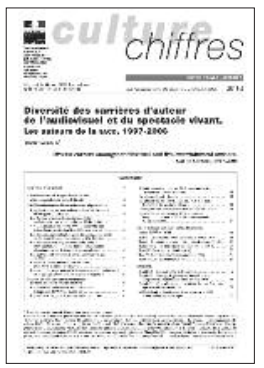

Marie Gouyon

Avril 2011, 32 pages

En 2008, la Société des auteurs et compositeurs dramatiques (SACD) a versé 136 millions d'euros de droits à 17700 auteurs et compositeurs dramatiques. Entre 1997 et 2008, les répertoires gérés par la sacd ont beaucoup évolué, en particulier celui de l'audiovisuel, du fait des mutations technologiques intervenues depuis la fin des années 1990 dans ce secteur : apparition puis généralisation de la télévision numérique terrestre (TNT), développement des chaînes thématiques, etc.

Les auteurs se distinguent nettement les uns des autres selon le répertoire (audiovisuel ou spectacle vivant) auquel ils participent, leur durée et leur volume de production, leur niveau de contributions aux diffusions et aux représentations, ainsi par les montants de droits perçus. La population des auteurs et compositeurs dramatiques reste âgée et très masculine, bien que les femmes y soient de plus en plus nombreuses.

\section{CC-2011-6}

\section{L'emploi salarié dans le spectacle en 2008 : une diversité de situations}

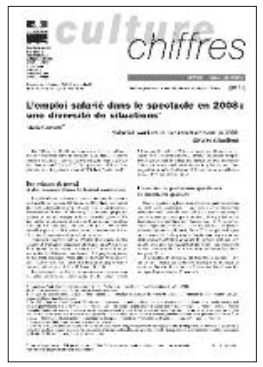

Marie Gouyon

Juin 2011, 12 pages

En 2008, 440000 personnes sur les 23 millions de salariés en France ont travaillé au moins une heure dans le secteur du spectacle (audiovisuel et spectacle vivant). Les volumes de travail et les rémunérations sont fortement concentrés par une minorité de salariés : $5 \%$ des salariés du spectacle perçoivent $30 \%$ du total des rémunérations. Polyvalence et pluriactivité caractérisent le salariat dans le spectacle, ce qui se traduit par une fragmentation de l'emploi : important recours aux contrats à durée déterminée et perception d'indemnités chômage en complément des salaires. Une typologie des salariés, articulée autour du volume de travail et du montant des salaires horaires, permet de caractériser six types principaux, des professionnels les plus impliqués, qui exercent au moins les trois quarts de leur activité dans le spectacle vivant (35\%) ou dans l'audiovisuel (37\%), aux salariés épisodiques (11\% dans l'audiovisuel et $17 \%$ dans le spectacle vivant). 
CC-2014-2

Tendances de l'emploi dans le spectacle

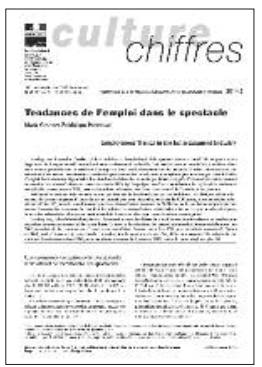

Marie Gouyon, Frédérique Patureau

Janvier 2014, 8 pages

En vingt ans, le nombre d'artistes et de techniciens de l'audiovisuel et du spectacle vivant a doublé. Portée par le développement de l'emploi salarié intermittent, cette croissance des effectifs s'est inscrite dans un marché du travail extrêmement dynamique en termes de créations d'entreprises (tout particulièrement dans le spectacle vivant) et de volume de travail offert. Pour autant, la croissance des effectifs professionnels s'est effectuée à un rythme plus soutenu que celui de l'offre d'emploi, instaurant une dégradation des situations individuelles mesurée par la baisse de près d'un quart du volume annuel de travail et des rémunérations au cours des années 1990. Après quelques années de stabilisation, la reprise de la croissance des effectifs renoue, depuis 2008, avec cette tendance de longue durée au resserrement de l'activité et des revenus.

Artistes et techniciens intermittents ne sont pas touchés de la même façon par ces évolutions. En 2009, un artiste intermittent déclare en moyenne 44 jours de travail annuel pour une rémunération brute de 9200 euros, et un technicien intermittent déclare 82 jours de travail annuel pour une rémunération moyenne de 16600 euros. Les artistes enregistrent donc des durées annuelles moyennes de travail et des volumes de rémunération très inférieurs à ceux des personnels techniques ; la part des indemnités chômage est aussi, de ce fait, beaucoup plus importante dans leur revenu global.

En vingt ans, la flexibilité de l'emploi s'est fortement accrue : les durées de travail se sont fractionnées en un nombre plus important de contrats de travail de courte durée. Là encore, les situations des artistes et des techniciens sont différentes : en 2009, un artiste déclare en moyenne 15 contrats de travail dans l'année contre 7 en 1990, pour une durée moyenne de 3 jours en 2009, contre 10 jours vingt ans plus tôt. Un technicien du spectacle, de son côté, déclare en moyenne 14 contrats de travail dans l'année contre 6 en 1990, pour une durée moyenne de 6 jours en 2009 , contre 14 jours vingt ans plus tôt.

\section{Rapport de l'Observatoire de l'égalité femmes/hommes dans la culture et la communication}

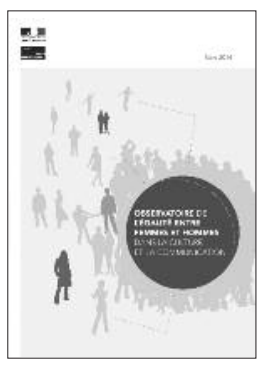

\section{Mars 2014}

Disponible sur www.culturecommunication.gouv.fr, rubrique «Égalité entre femmes et hommes »

\section{Tous les documents publiés par le DEPs sont téléchargeables sur http://www.culturecommunication.gouv.fr/Etudes-et-statistiques et sur http://www.cairn.info}

Le DEPS n'assurant pas de diffusion physique de ses collections, nous vous proposons de vous informer régulièrement des parutions par message électronique.

Pour ce faire, merci de bien vouloir nous communiquer votre courriel à l'adresse contact.deps@culture.gouv.fr 
En 20II, les femmes représentent $29 \%$ des auteurs de la SACD et reçoivent $24 \%$ des droits versés. Une femme perçoit ainsi, en 20II, un revenu moyen inférieur de $24 \%$ à celui d'un homme.

Les écarts de droits versés entre femmes et hommes trouvent plusieurs éléments d'explication : les femmes participent à moins d'œuvres que les hommes, en particulier dans le cinéma, le théâtre et les arts de la rue. Elles n'exercent pas non plus les mêmes fonctions, et sont ainsi plus souvent auteurs de texte ou chorégraphes que réalisatrices ou compositrices. À discipline et fonction égales, les écarts de rémunération sont pratiquement inexistants dans la radio et la télévision, mais subsistent dans le cinéma et le spectacle vivant : ces écarts persistants peuvent être liés à d'autres facteurs comme, par exemple, une fréquentation des spectacles ou une programmation des œuvres moins importante, dans des lieux de représentation ou sur des canaux de diffusion différents et à des horaires moins favorables. 